\title{
Evolution of an eruptive flare loop system
}

\author{
P. Romano ${ }^{1}$, F. Zuccarello ${ }^{2}$, L. Fletcher ${ }^{3}$, F. Rubio da Costa ${ }^{2,3}$, H. M. Bain ${ }^{3}$, and L. Contarino ${ }^{2}$ \\ 1 INAF - Osservatorio Astrofisico di Catania, via S. Sofia 78, 95123 Catania, Italy \\ e-mail: prom@oact.inaf.it \\ 2 Dipartimento di Fisica e Astronomia - Sezione Astrofisica, Università di Catania, via S. Sofia 78, 95123 Catania, Italy \\ 3 Department of Physics and Astronomy, University of Glasgow, Glasgow G12 8QQ, UK
}

Received 7 November 2008 / Accepted 13 March 2009

\section{ABSTRACT}

\begin{abstract}
Context. Flares, eruptive prominences and coronal mass ejections are phenomena where magnetic reconnection plays an important role. However, the location and the rate of the reconnection, as well as the mechanisms of particle interaction with ambient and chromospheric plasma are still unclear.

Aims. In order to contribute to the comprehension of the above mentioned processes we studied the evolution of the eruptive flare loop system in an active region where a flare, a prominence eruption and a CME occurred on August 24, 2002.

Methods. We measured the rate of expansion of the flare loop arcade using TRACE $195 \AA$ images and determined the rising velocity and the evolution of the low and high energy hard X-ray sources using RHESSI data. We also fitted HXR spectra and considered the radio emission at 17 and $34 \mathrm{GHZ}$.

Results. We observed that the top of the eruptive flare loop system initially rises with a linear behavior and then, after $120 \mathrm{mn}$ from the start of the event registered by GOES at 1-8 $\AA$, it slows down. We also observed that the heating source (low energy X-ray) rises faster than the top of the loops at $195 \AA$ and that the high energy X-ray emission $(30-40 \mathrm{keV})$ changes in time, changing from footpoint emission at the very onset of the flare to being coincident during the flare peak with the whole flare loop arcade.

Conclusions. The evolution of the loop system and of the X-ray sources allowed us to interpret this event in the framework of the Lin \& Forbes model (2000), where the absolute rate of reconnection decreases when the current sheet is located at an altitude where the Alfvén speed decreases with height. We estimated that the lower limit for the altitude of the current sheet is $6 \times 10^{4} \mathrm{~km}$. Moreover, we interpreted the unusual variation of the high energy HXR emission as a manifestation of the non thermal coronal thick-target process which appears during the flare in a manner consistent with the inferred increase in coronal column density.
\end{abstract}

Key words. Sun: activity - Sun: flares - Sun: magnetic fields

\section{Introduction}

During most flares it is possible to observe in $\mathrm{H} \alpha$ bright ribbons, which usually appear in pairs, moving away from one another at a speed that can be initially $\sim 100 \mathrm{~km} \mathrm{~s}^{-1}$ decreasing to less than $1 \mathrm{~km} \mathrm{~s}^{-1}$ over some hours. Accompanying the flare ribbons is a system of flare-loops which initially appears at low altitude and then moves upward into the corona in consort with the motion of the ribbons Moore et al. (1980). These loops are commonly called post-flare loops (however, it has been recently suggested by Svestka 2007 that this term should be avoided because they are parts of the flare itself) or flare loop system. They are usually visible in EUV lines or in the X-ray range during the main phase and are the manifestation of the reconfiguration of the magnetic field lines after the disruption of the coronal field.

Recently, a better understanding of the phenomena related to solar eruptions has allowed us to realize that a catastrophic loss of mechanical equilibrium in a particular coronal magnetic configuration can trigger the reconnection, which accounts for flare ribbons and flare-loops (Forbes \& Priest 1995). In this scenario the reconnection of stretched-out field lines occurs, with the reconnection point rising and new loops continually forming and cooling (Kopp \& Pneuman 1976). A series of simulations have also been performed to deal with the loss of equilibrium and the formation of current sheets (Amari et al. 2000; Roussev et al. 2003; Török \& Kliem 2005). However, these models do not reproduce the continual downflow of material observed in the loop system during its lifetime. For example, the total mass flowing downward during a whole flare of $3 \mathrm{~b}$ class is of the order of $10^{16} \mathrm{~g}$, corresponding to the total mass of the whole corona (Pneuman 1981). This material must somehow be supplied from the chromosphere. To account for this invisible upflow, the process of chromospheric evaporation was suggested by many authors (see Antiochos \& Sturrock 1978, and references therein). In the usual picture of a flare, the chromospheric heating source to drive evaporation originates with non-thermal particles accelerated during the flare impulsive phase, but during the gradual phase Cargill \& Priest (1982) proposed also a dynamic model describing the main phase of the flare with field lines closing down by reconnection and a pair of slow MHD shocks, of the type proposed by Petschek, propagating down the legs of the loops. These shocks could heat the plasma to $30 \mathrm{MK}$ or more. From numerical experiments Forbes \& Priest (1983) realized that a fast-mode termination shock exists below the down-flowing jet of plasma from the reconnection region, since it is slowed and diverted sideways by the obstacle presented by the closed field lines. Then Forbes \& Malherbe (1986) split each slow mode shock into an isothermal slow shock and a standing conduction front that propagates down to the chromosphere and drives evaporation from outside edges of the $\mathrm{H} \alpha$ ribbons. In this scenario the soft X-rays come from the hot loops that are heated by the conduction front and the hard X-rays come partly from the 


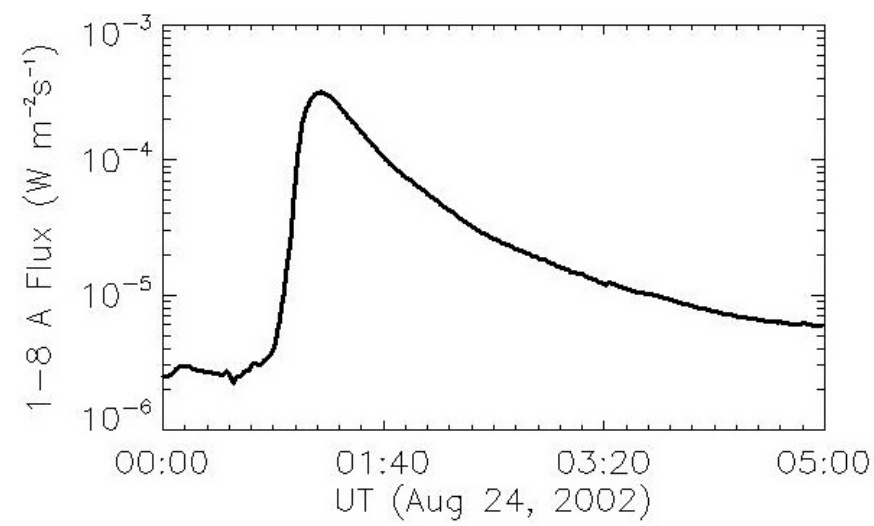

Fig. 1. GOES X-ray flux vs. time. The beginning of the time interval is 00:00 UT on Aug. 24, 2002.

footpoints where the fast particles and conduction fronts reach the chromosphere and partly from the downflow jet heated by the slow shocks and the denser plasma heated and compressed by fast shocks.

The reconnection process affects the acceleration of energetic particles along the flare loops and of ejected plasma into interplanetary space. In order to provide a further observational contribution to its understanding we studied an X-class flare which occurred at the West limb of the Sun using TRACE images at $195 \AA$, RHESSI data in the low and high energy hard $\mathrm{X}$-rays and Nobeyama radioheliograph data at 17 and $34 \mathrm{GHz}$. The particular location of the flare allowed us to measure the geometrical parameters of the eruptive flare loop system and the height of the heating source. We also determined the evolution of the hard X-ray bremsstrahlung emission along the loops and the brightness temperature at 17 and $34 \mathrm{GHz}$.

The layout of the present paper is as follows: in the next section we present the data and the analysis methods. In Sect. 3 we describe our results and in Sect. 4 we give our conclusions.

\section{Data analysis}

On Aug. 24, 2002 GOES (Garcia 1994) observed a flare of X3.1 class in X-rays at 1-8 $\AA$ in the active region NOAA 10069. This event started at 00:49 UT and ended at 4:59 UT, while the maximum emission was reached at 1:12 UT (Fig. 1).

In the same active region the IPS - Learmonth Observatory (Kennewell \& Cornelius 1983) registered a prominence eruption starting at 00:55 UT. Moreover, SOHO/LASCO C2 and C3 (Brueckner et al. 1995) observed a coronal mass ejection at the West limb with a linear velocity reported in the LASCO catalogue (http: //cdaw.gsfc.nasa.gov/CME_list/) of about $1913 \mathrm{~km} \mathrm{~s}^{-1}$ since 1:23 UT to 3:21 UT. Because of its particular location (at the limb) and its peculiar orientation (with the line connecting the footpoints approximately perpendicular to the equator) the flare-loop system observed during the main phase of the flare allowed us to study the evolution of some physical parameters and to compare them with those provided by models. We observed the loop system in the EUV range using TRACE (Handy et al. 1999) images at $195 \AA$, in X-rays by RHESSI (Lin et al. 2002) in the $3-6 \mathrm{keV}, 6-12 \mathrm{keV}, 12-25 \mathrm{keV}$ and $30-80 \mathrm{keV}$ bands and in the radio band, at 17 and $34 \mathrm{GHz}$.

The images obtained at $195 \AA$ by TRACE from 00:00 to $04: 59$ UT cover a field of view of $1024 \times 1024$ pixels $^{2}$ $\left(371 \times 371 \mathrm{Mm}^{2}\right)$, with a spatial and a temporal resolution of 1 arcsec and $\sim 60 \mathrm{~s}$, respectively. We corrected TRACE data for instrumental effects, like pedestal and dark current, and for readout noise. A further correction was done for hot pixels, caused by electrons which are not properly flushed out after the CCD readout, and for radiation spikes due to cosmic rays. The data were then normalised for the different exposure times.

To obtain the RHESSI images we used the CLEAN method (Hurford et al. 2002) with an integration interval of $4 \mathrm{~s}$ for the $3-6,6-12$ and $12-25 \mathrm{keV}$ bands and of $28 \mathrm{~s}$ for the $30-40 \mathrm{keV}$ band. We used data taken from detectors 1-9, obtaining a spatial resolution of 4 arcsec. The application of the different standard techniques of back projection, MEM, MEMVIS, Pixon and forward-fitting (see Hurford et al. 2002), produced similar results, characterized by the same location of the brightening during the flare peak. Both TRACE and RHESSI data have been corrected using the standard SolarSoft routines.

The event was observed also by the Nobeyama Radioheliograph (Nikajima et al. 2004) at 17 and 34 GHz. The brightness temperature at 17 and $34 \mathrm{GHz}$ and the polarization degree images at $17 \mathrm{GHz}$ have provided further information about the emission mechanism of the particles from the reconnection site (see Karlický 2004).

The co-alignment among the data of TRACE, RHESSI and Nobeyama has been made taking into account the fits header information. We resized the images of RHESSI and Nobeyama to obtain the same pixel resolution of TRACE, then we aligned them considering the latitude and longitude coordinates of the field of view centers with respect to the Sun center. From the comparison among different limb locations we have measured a co-alignment uncertainty of the order of 4-5 arcsec.

\section{Results}

At the beginning of our TRACE dataset we observed the presence of an EUV filament channel, i.e. a dark elongated region, just over the limb, probably corresponding to a small prominence and indicated by an arrow in Fig. 2a. This structure remained stable till 00:52 UT, when it showed ejection of plasma (Fig. 2b) at the same time of the registered prominence eruption. At that time the high energy HXR emission at $30-40 \mathrm{keV}$ was concentrated in the southest loop footpoint (see the white contour in Fig. 2b). We also noted that the radio brightness temperature at $17 \mathrm{GHz}$ showed a clear emission at $1 \mathrm{MK}$ in the same footpoint (see the grey contour in Fig. 2b).

From 00:54 UT till the end of TRACE sequence (4:59 UT) we see the eruptive flare-loop system becoming larger and larger (see Figs. 2c,d). We measured the increasing of the height $p$ of the loops and of the distance $2 \lambda$ between the footpoints (see Figs. 3a,b, respectively). We determined the top of the loop system considering the biggest loop in each frame and localizing the maximum of the gradient of the intensity signal along the perpendicular to the limb. We determined the location of the footpoints using an analogous method, but considering the gradient along the limb at the bottom of the arcade. We assumed that the flare-loops were perpendicular to the solar surface and we neglected the uncertainty coming from their different inclination. Therefore, taking into account that the only source of error is due to the location of the maximum of gradient we can assume that the uncertainties on the determination of $p$ and $2 \lambda$ correspond to the resolution of TRACE, i.e. 1 arcsec. When the loop arcade appeared (00:54 UT), we determined for these parameters the following values: $p \sim 2.6 \times 10^{4} \mathrm{~km}$ and $2 \lambda \sim 3.8 \times 10^{4} \mathrm{~km}$. At the end of our observing dataset $p$ was $\sim 7.5 \times 10^{4} \mathrm{~km}$ and $2 \lambda$ was $\sim 8.7 \times 10^{4} \mathrm{~km}$, corresponding to a less squeezed shape of the loops. These results are in agreement with those obtained by 


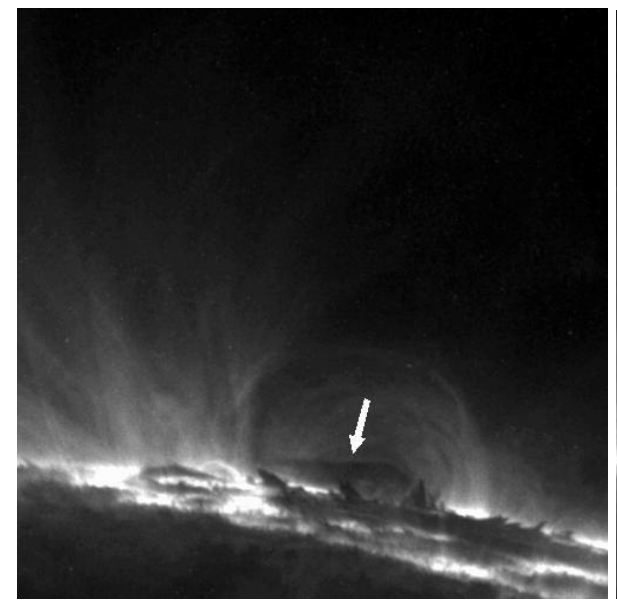

(a) 00:15 UT

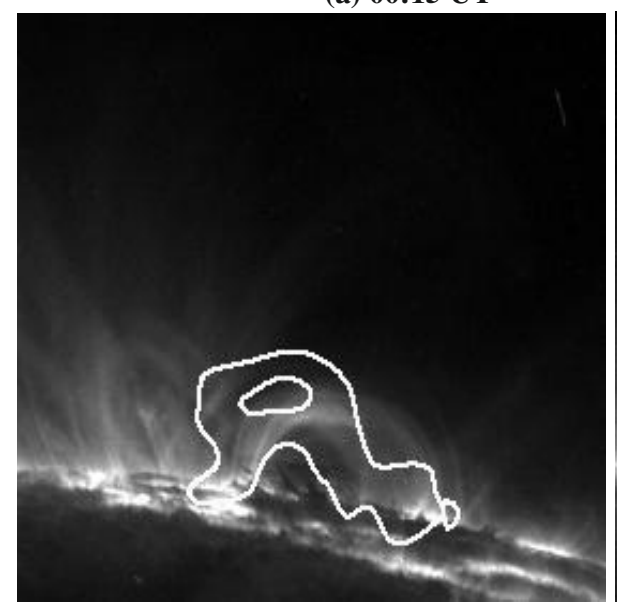

(c) 00:56 UT

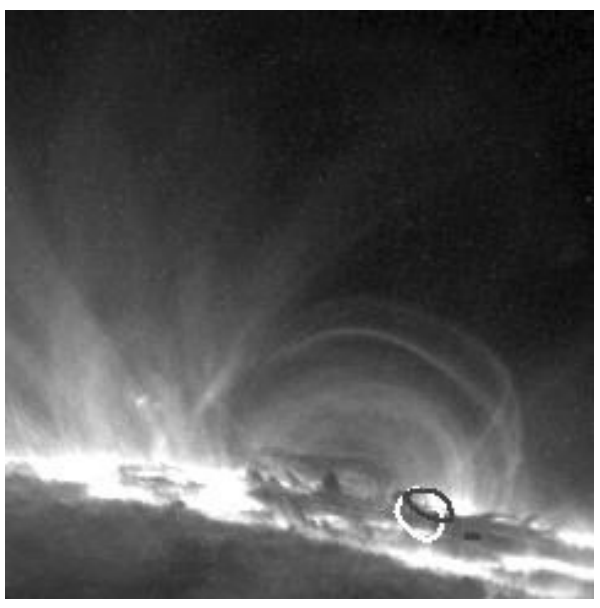

(b) 00:52 UT

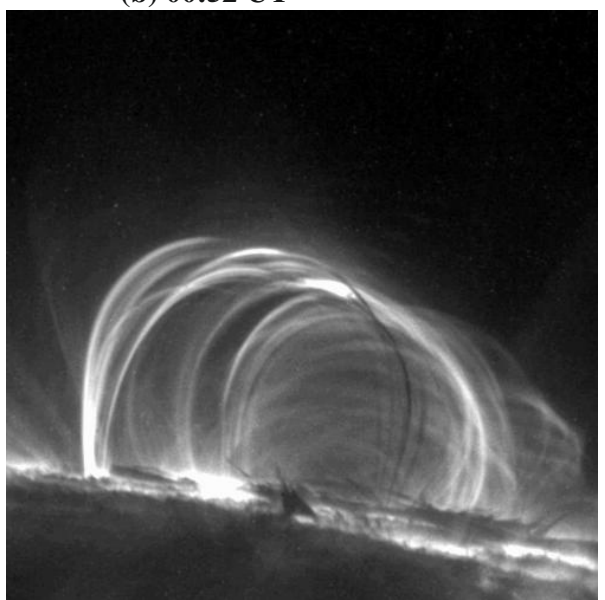

(d) 04:59 UT

Fig. 2. Sequence of TRACE images acquired at $195 \AA$ during an X3.1 flare. In a) the arrow indicates the presence of the EUV filament channel which shows a destabilization at 00:52 UT. In b) the white and gray contours indicate 50\% of the peak flux at $30-40 \mathrm{keV}$ and $1 \mathrm{MK}$ of the $17 \mathrm{GHz}$ brightness temperature, respectively. In c) the contours indicate $20 \%$ and $70 \%$ of the flux peak at $30-40 \mathrm{keV}$. The field of view is $\sim 180 \times 180 \mathrm{Mm}^{2}$. North is at the left and West on the top.

Noglik et al. (2005), who showed that in a similar event occurred on March 18, 1999, the apex of the loops expanded more rapidly than the footpoints separation.

We also determined the rising velocity of the top of the involved eruptive flare loops. We measured this quantity with a sampling of $p$ of $20 \mathrm{mn}$. Figure $3 \mathrm{c}$ shows that the maximum velocity of $\sim 6 \pm 0.4 \mathrm{~km} \mathrm{~s}^{-1}$ is reached at $\sim 2: 50 \mathrm{UT}$, i.e. after $\sim 120 \mathrm{mn}$ from the start of the event registered by GOES at 1$8 \AA$. This velocity is a factor three greater than the value of the looptop speed measured by Noglik et al. (2005). After 2:50 UT a slowing down occurred, corresponding to a slower rate of reconnection.

From RHESSI data we deduced the location of the low energy X-ray source, which is always located above the top of the loop system observed by TRACE. In Figs. 4a,b we show contours of $50 \%$ and $70 \%$ of the flux peak at $12-25 \mathrm{keV}$ superposed on TRACE loop images. Analogous results are obtained for 36 and $6-12 \mathrm{keV}$ bands. The height $h$ of the baricenter of the low-energy X-ray source at $12-25 \mathrm{keV}$ vs. time is reported in Fig. 3d. We note that $h$ reaches $\sim 8.5 \times 10^{4} \mathrm{~km}$ in only $100 \mathrm{mn}$ since 00:50 UT. This value corresponds to an average rising velocity of $\sim 10 \mathrm{~km} \mathrm{~s}^{-1}$, which is higher than the velocity of the top of the loops observed at $195 \AA$.

During the peak and the main phase of the flare the high energy HXR at 30-40 keV and the radio emissions showed a quite similar behavior. They started to increase at 00:52 UT. The HXR at 30-40 keV reached its maximum at 00:56 UT when the $20 \%$ of the flux peak covered the whole loop arcade and the $70 \%$ was located at the top of the loops (Fig. 2c).

The radio emission reached its maximum at 01:00 UT $\left(2.29 \times 10^{7} \mathrm{Jy}\right.$ at $17 \mathrm{GHz}$ and $3.9 \times 10^{6} \mathrm{Jy}$ at $\left.34 \mathrm{GHz}\right)$. As reported by Karlický (2004) at about 01:12 the flux spectrum $F_{v} \propto v^{\alpha}$ had positive spectral index $\alpha$, corresponding to optically-thick emission, while at about 01:22 UT, i.e. well after the flare maximum, a region appeared at the top of the flare loop with $\alpha$ less than zero, and started to increase in size. Moreover, at 01:30 UT two strong sources with a minimum value of $\alpha \sim-2.5$ were located near the footpoints. A negative spectral index at these frequencies corresponds, as stated by Karlický (2004), to gyrosynchtrotron emission from non-thermal electrons. The suggestion is that radio emission provides evidence for the presence of accelerated electrons late in the flare. However, it is worth noting that RHESSI images in higher energy bands $(40-50 \mathrm{keV}$ or 50-80 keV) do not show any well - defined emission along the loop arcade. The absence of such RHESSI emission limits the maximum energy to which the late-phase electrons are accelerated to maybe $30 \mathrm{keV}$.

Figure 5 shows RHESSI X-ray spectra for times before and after the flare peak using front detectors 1, 3, 4, 6, 8 and 9. Energy bins of $0.33 \mathrm{keV}$ and $1 \mathrm{keV}$ were used below and above $12 \mathrm{keV}$, 

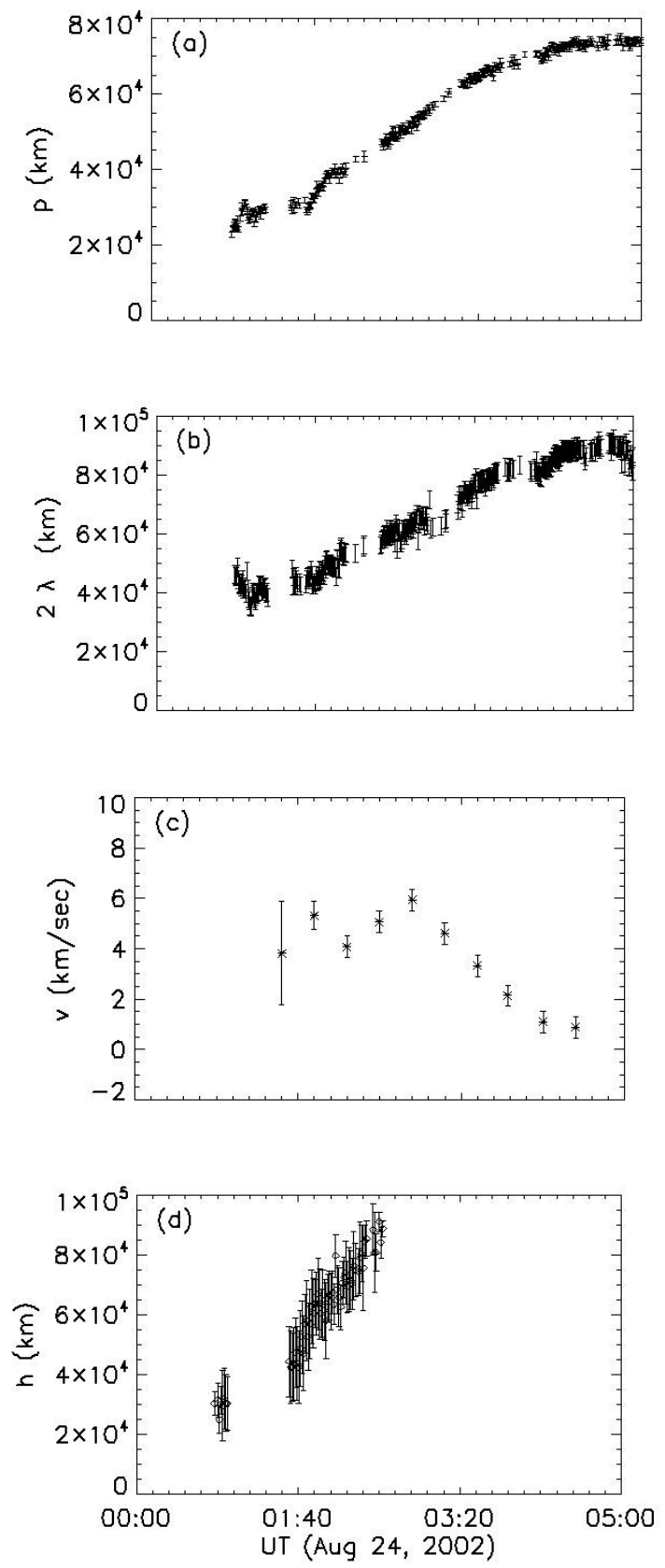

Fig. 3. a) Height of the top of the flare loop system vs. time; b) distance between the footpoints vs. time; c) velocity of the height of the involved eruptive flare loops vs. time; d) Height of the low energy X-ray source baricenter vs. time.

respectively. The black lines show the noise-subtracted datapoints, the grey lines show the level of the background. We fitted the spectra with an isothermal component (green lines), characterized by a temperature reported in each plot, and with a broken power law component (blue lines) which have a fixed spectral index of 1.5 below the power law break energy. A freely varying spectral index then fits the higher energy datapoints above break energy and is reported in each plot. Note that for times 01:58 and 02:30 the data is fitted with a thermal component only. An additional Gaussian line (orange) was added to fit the line feature seen at $11 \mathrm{keV}$. It is thought that this is an instrumental feature but its origin is not fully understood. The red lines show the overall fits. From the fits we can see that at the time of the flare peak (around 00:56) the event is thermally dominated up to about

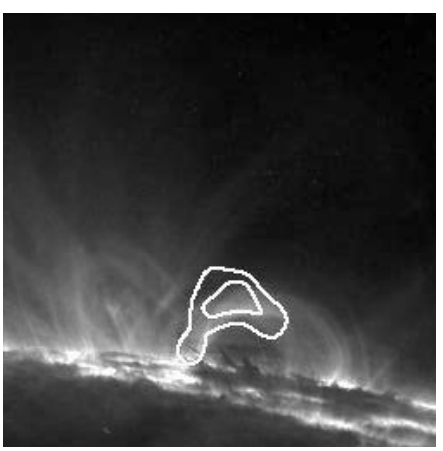

(a) 00:56 UT

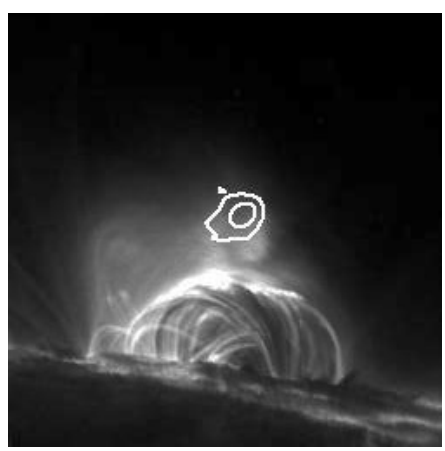

(b) 01:58 UT
Fig. 4. Contours of $50 \%$ and $70 \%$ of the flux peak at $12-25 \mathrm{keV}$ overposed on TRACE images taken at $195 \AA$.

25-30 keV and non-thermal above this energy. Therefore, the contours shown in Fig. 2c are dominated by non-thermal emission from the corona.

Using the method of Veronig \& Brown (2004) we also computed the temporal variation of the loop column density $N$. We used TRACE images to define the volume $V=A L=\pi A \lambda$, where $A$ and $\lambda$ are the footpoint area and semi-distance, respectively, and $L$ is the loop length. Under the assumption of cylindrical volume of the loops and estimating an average radius of the footpoint of 5 arcsec, we obtain a value of $A$ of the same order of Veronig \& Brown (2004) events, i.e. $10^{17} \mathrm{~cm}^{2}$. The mean loop plasma electron number density is then $n_{\mathrm{e}}=(E M / V)^{1 / 2}$ (for a filling factor 1), and the loop leg column number density $N=n_{\mathrm{e}} L / 2$, where EM is the emission measure. By assuming that GOES is observing emission from the same (presumably multi-thermal) loops as are seen by TRACE and RHESSI at low energy we can use the GOES broadband response in the two channels at $0.5-4.0 \AA$ and $1.0-8.0 \AA$ to estimate the plasma EM, and hence calculate $\mathrm{N}(\mathrm{t})$. Figure 6 shows the evolution of $n_{\mathrm{e}}(\mathrm{t})$ and of $\mathrm{N}(\mathrm{t})$ during the event. We note that $n_{\mathrm{e}}$ reaches $3.5 \times 10^{11} \mathrm{~cm}^{-3}$ at 01:10 UT, while $N$ varies from from $0.5 \times 10^{20}$ to $7 \times 10^{20} \mathrm{~cm}^{-2}$ between 00:50 and 01:10 UT and then it decreases more smoothly in the subsequent period. The enhanced coronal column depth provides a collisional target to coronal electrons, such that all electrons with energy $E$ such that $E^{2} \leq 3 K \Lambda N$ are collisionally stopped (Veronig \& Brown 2004). Here $K=2 \pi \mathrm{e}^{4}, e$ being the charge of the electron, and $\Lambda$ the Coulomb logarithm. The energy of electrons that would be collisionally stopped in the loop half length (assuming acceleration in the midpoint of the loop) corona thus varies from $\sim 20-50 \mathrm{keV}$. This can account for the predominance of coronal HXR emission.

\section{Discussion and conclusions}

This event gives some indirect information on flare reconnection processes and provides useful information to better understand the behavior of energetic particles during this kind of events. It shows evidence from X-rays and radio of coronal electrons with energies of a few tens of $\mathrm{keV}$, far into the flare decay phase.

We could divide the event into two phases: - phase 1 (00:52-00:56 UT), characterized by the radio emission at $17 \mathrm{GHz}$ and the X-ray emission at $30-40 \mathrm{keV}$ in the southern footpoint, the destabilization of the EUV filament channel and the prominence eruption; - phase 2 (00:56-04:59 UT), characterized by the expansion of the flare loop system, the upward motion of the baricenter of the low energy X-ray source and a 


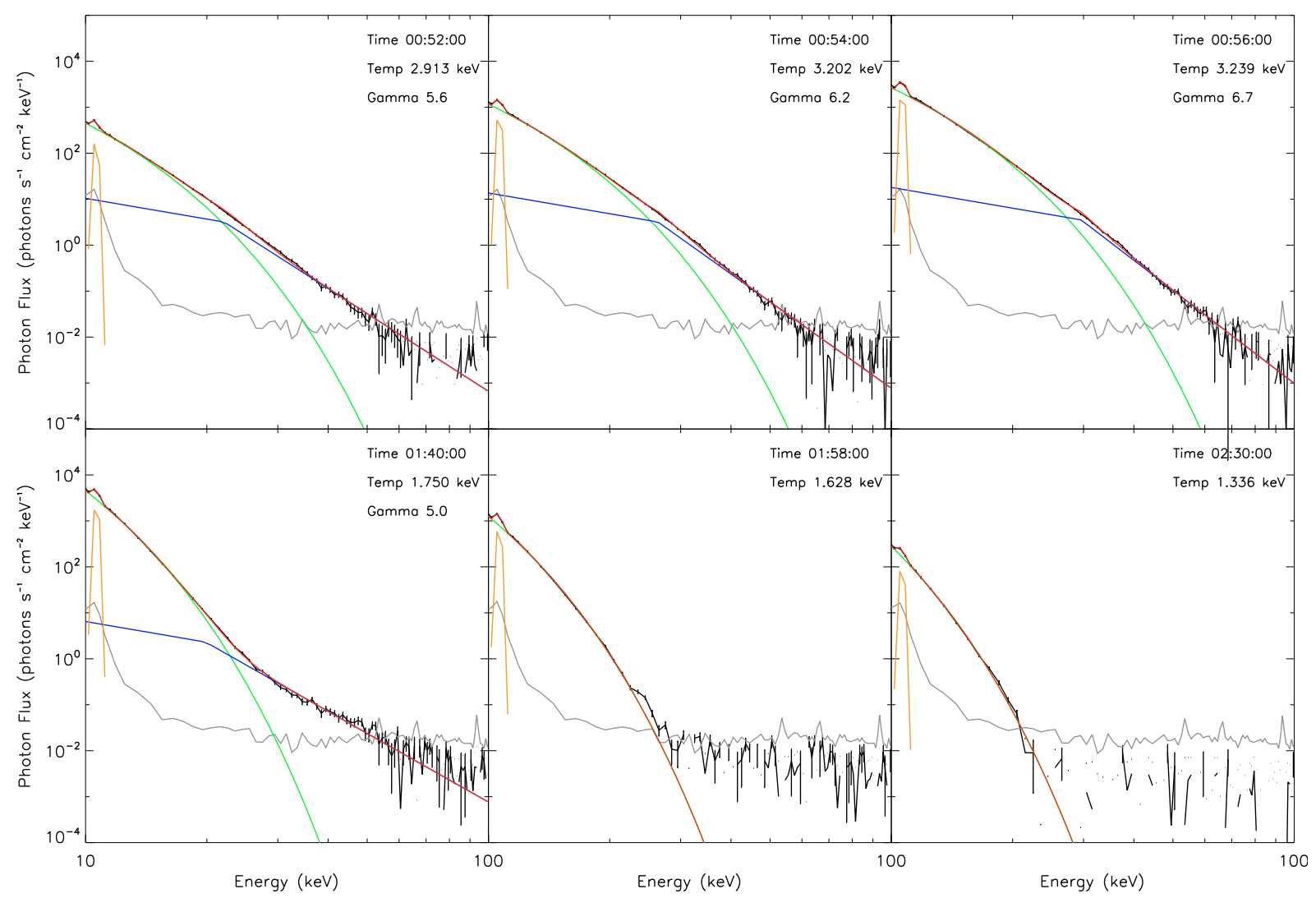

Fig. 5. RHESSI spectra around the flare peak time. The black and grey lines show the noise-subtracted datapoints and the level of the background, respectively. We fitted the spectra with an isothermal component (green lines) characterized by a temperature reported in each plot, and with a broken power law component (blue lines), characterized by a fixed spectral index of 1.5 below the power law break energy. An additional Gaussian line (orange) was added to fit the line feature seen at $11 \mathrm{keV}$. The red lines are the overall fits.

region with spectral index $\alpha<0$ at the top of the flare loop system and at its footpoints, as reported by Karlický (2004).

In the early phase, the activity is concentrated in the lower atmosphere, and is closely-associated in time with the ejection of a filament. A reasonable scenario is that the loss of stability of the filament leads to its ejection, disrupting the overlying field. There are several different models for the loss of stability of the filament - e.g. tether-cutting (Sturrock et al. 2001; Moore et al. 2001) or an MHD instability - e.g. a kink-mode instability (e.g. Török \& Kliem 2005). Reconnection below the ejecting filament allows stored magnetic energy to be converted into kinetic and thermal energies which account simultaneously for both the mass ejection and the intense heating. The ongoing energisation well into the flare gradual phase suggests that the coronal magnetic field is still relaxing and reconnecting long after the filament has departed.

In the second phase of our event, a model involving the slow evolution of a large-scale current sheet may find application, and we interpret our observations in this context, focussing in particular on a two-dimensional model of an ejecting flux rope proposed by Lin \& Forbes (2000). This analytical model provides a framework for relating the motion of the ejected material and the evolution of the coronal loops structure produced by reconnection to the reconnection rate as described by the Alfvén Mach number at inflow, $M_{\mathrm{A}}$. The model examines (among other things) the variation of the measurable quantity $p$ (the height of the arcade of post-flare closed loops) as a function of time under different conditions. Our measurement of $p$ shows an approximately monotonic increase of height with time, with deceleration setting in around 160-180 $\mathrm{mn}$ after the appearance of the loops in TRACE, when it reached $\sim 6 \times 10^{4} \mathrm{~km}$. In the context of the Lin \& Forbes model, this would correspond to a reduction in the absolute rate of coronal reconnection as the reconnection region moves into a higher coronal regions where the Alfvén speed is decreasing with height. Lin \& Forbes find that if $M_{\mathrm{A}}$ exceeds 0.041 then no such deceleration will occur, allowing us to infer that $M_{\mathrm{A}} \leq 0.04$ in the framework of their model. Observationally, the deceleration of $p$ sets in later than in the Lin $\&$ Forbes case with $M_{\mathrm{A}}=0.01$, from which we infer $M_{\mathrm{A}} \geq 0.01$.

The increase of the looptop height observed by TRACE is consistent with the slow upward evolution of a post-reconnection structure. However, a rising low-energy HXR source has been interpreted by previous authors as having a closer connection with the current sheet itself, indicating the reconnection outflow close to the lower end of a rising current sheet (e.g. Sui \& Holman 2003). These hot closed loops will then cool to lower energies, appearing later in TRACE (e.g. Gallagher et al. 2002). The baricenter of the low energy X-ray source in this event is moving upwards at a higher speed than the post-flare loops, at $\sim 10 \mathrm{~km} \mathrm{~s}^{-1}$ compared to $\sim 5 \mathrm{~km} \mathrm{~s}^{-1}$ for the TRACE loops. The X-ray source speed is equal to the value measured by Gallagher et al. (2002), who considered the centroids of the X-ray source at 3-25 keV for an X1.5 flare occurred on April 21, 2002. Lin \& Forbes discuss whether a difference in the slow rise rate of closed post-flare $\mathrm{X}$-ray arcades and the rapid rise of giant X-ray arches (the top of which they also associate with the lower end of a rising current 


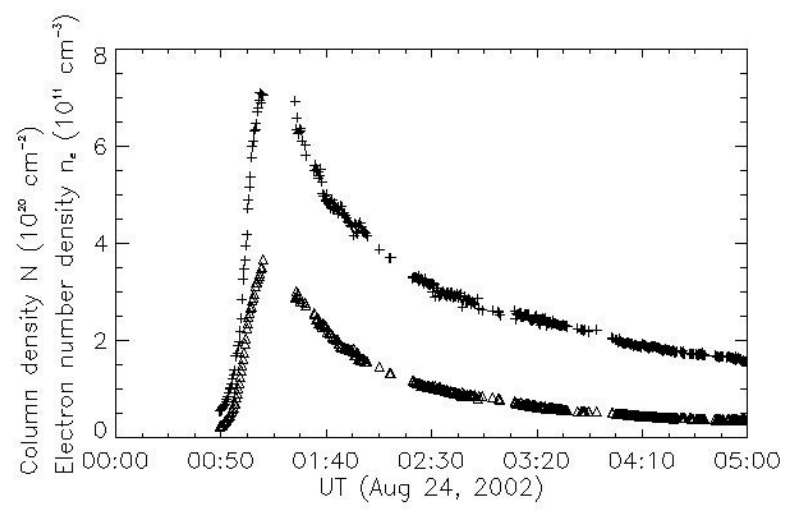

Fig. 6. Time history of electron number density $n_{\mathrm{e}}$ (triangles) and loop column density $N$ (crosses). The beginning of the time interval is 00:00 UT on Aug. 24, 2002.

sheet) can be explained within their scenario. Their conclusion is that an Alfvén speed varying with height, as in their model, permits such a behavior, so that the entire post-eruption arcade becomes stretched.

This is somewhat less clear in our case, because the temperatures sampled by the RHESSI source and the TRACE looptops are different (with TRACE detecting cooler plasmas than RHESSI) thus there is a time lag between sources appearing in RHESSI and in TRACE. If we interpret the TRACE loops as cooled loops that were previously seen in RHESSI, and have retracted back following reconnection, then the apparent slowing of their growth - while the RHESSI source proceeds at a roughly constant upward rate - could merely reflect the change in the cooling timescale as the event proceeds, and no actual stretching of the arcade. In this event we are able to estimate the cooling timescales to try and decide this point.

At the beginning and the end of the rising HXR source observations: at 01:40 UT and at 02:30 UT we can estimate the loop density as $2 \times 10^{11} \mathrm{~cm}^{-3}$ and $1 \times 10^{11} \mathrm{~cm}^{-3}$ respectively (see Fig. 6). These are likely to be overestimates, since they are arrived at using the GOES emission measure and the dimensions of the TRACE loops, which are shorter than the loops where the RHESSI source is found. As an estimate for the loop length $L$ we take the height of the HXR source, i.e. $4.5 \times 10^{4} \mathrm{~km}$ and $9.0 \times 10^{4} \mathrm{~km}$ at 01:40 UT and at 02:30 UT respectively. The time scale for cooling by unsaturated heat flux is (Aschwanden 2004, Ch. 16):

$\tau_{\text {cond }}=\frac{21}{5} n_{\mathrm{e}} \frac{k L^{2}}{\kappa T^{5 / 2}}$,

where $n_{\mathrm{e}}$ is the electron number density, $k$ is Boltzmann's constant, $L$ is the loop length, $\kappa=9.2 \times 10^{-7} \mathrm{ergs} \mathrm{s}^{-1} \mathrm{~cm}^{-1} \mathrm{~K}^{-7 / 2}$ is the coefficient of classical (Spitzer) thermal conduction and $T$ is the temperature of the cooling plasma. The radiative loss time is:

$\tau_{\text {rad }}=\frac{3 k T}{n_{\mathrm{e}} \Lambda(T)}$

where $\Lambda(T)$ is the radiative loss rate at $20 \mathrm{MK}$ which - depending primarily on the assumed element abundance - is one to a few $\times 10^{-23} \mathrm{erg} \mathrm{s}^{-1} \mathrm{~cm}^{-3}$ for temperatures on the order of $10 \mathrm{MK}$ as we have here (see compilation in Aschwanden, 2004, Ch. 2). We shall use $\Lambda(T)=3 \times 10^{-23} \mathrm{ergs} \mathrm{s}^{-1} \mathrm{~cm}^{-3}$.

Thus, the conductive cooling time, assuming a constant maximum value of $T \sim 25 \mathrm{MK}$, varies from $700 \mathrm{~s}$ at $01.40 \mathrm{UT}$ to $1400 \mathrm{~s}$ at $02.30 \mathrm{UT}$. These are upper limits, because of the likelihood that we have overestimated the density where the RHESSI source is formed. Again assuming a constant value for $T \sim 25 \mathrm{MK}$ the radiative cooling time is around $5000 \mathrm{~s}$ at $01.40 \mathrm{UT}$ and $10000 \mathrm{~s}$ at $02.30 \mathrm{UT}$. These are lower limits, again because of the density estimate being an upper limit. We conclude that conduction dominates the initial (i.e. from high temperatures) cooling of this event. The cooling time increases by a factor 2 through the event, thus as the RHESSI source cools into TRACE loops it takes longer for the post-reconnection loops to appear, during which time they can retract more. However, the difference between the HXR source position at 01.40 UT and the top of the TRACE loops at $01.40 \mathrm{UT}+700$ s (i.e. adding the cooling time) is $\sim 5 \times 10^{3} \mathrm{~km}$, whereas the difference between the HXR source position at $02.30 \mathrm{UT}$ and the top of the TRACE loops at $02: 30 \mathrm{UT}+1400 \mathrm{~s}$ is $\sim 3.5 \times 10^{4} \mathrm{~km}$. So unless the average retraction speed of the loops increases with time by a factor $\sim 3.5$ it seems likely that the increasing distance between RHESSI and TRACE loops does represent primarily an overall vertical stretching of the flare arcade, as in the Lin and Forbes model.

Using an approximate value of $1000 \mathrm{~s}$ for the time taken for a typical hot loop to disappear from RHESSI and reappear in TRACE, and an approximate value of $2 \times 10^{4} \mathrm{~km}$ for the RHESSI source and TRACE loop separation, gives a loop retraction speed of around $20 \mathrm{~km} \mathrm{~s}^{-2}$. This is an order of magnitude less than the speeds of the supra-arcade downflows observed in the late phase of some long duration flares by e.g. McKenzie \& Hudson (1999) and Sheeley et al. (2004), and generally interpreted as the retraction of reconnected loops at some fraction of the local Alfvén speed. A lower loop density, as we have argued above, would bring the two estimates more into agreement.

Comparing Figs. 3a,c we note that the speed of the baricenter of the low energy X-ray source may start to decrease at the end of RHESSI observation, i.e. $\sim 30 \mathrm{mn}$ earlier than $p$ starts to slow. This would imply that the current sheet length starts to decrease $\sim 100 \mathrm{mn}$ after the start of the eruption, as in the model of Lin \& Forbes for the Alfvén Mach number equal to 0.01 in an isothermal atmosphere (i.e. an exponential decreasing density with height).

Moreover, from RHESSI observations in the $30-40 \mathrm{keV}$ interval we note that after few minutes from the start of the eruption and during the impulsive phase of the flare, the X-ray emission does not coincide with the loop footpoints, as expected by the bremsstrahlung effect in the lower and higher density atmosphere, but with the whole loop length (Fig. 2c). We think that this unusual HXR emission can be interpreted as a manifestation of the non thermal corona thick-target process (Veronig \& Brown 2004). This implies that the coronal density has increased at an early stage in the flares (before the peak flux in $\mathrm{X}$-rays) possibly due to an evaporation process.

Acknowledgements. This work was supported by the European Commission through the SOLAIRE Network (MTRN-CT-2006-035484), by the Istituto Nazionale di Astrofisica (INAF), by the Agenzia Spaziale Italiana (contract I/015/07/0) and by the Università degli Studi di Catania. L.F. and H.M.B. are pleased to acknowledge the support of the UK Science and Technology Facilities Council via Rolling Grant ST/F002637, and a Ph.D. studentship.

\section{References}

Amari, T., Luciani, J. F., Mikic, Z., \& Linker, J. 2000, ApJ, 529, L49

Antiochos, S. K., \& Sturrock, P. A. 1978, ApJ, 354, 343

Aschwanden, M. J. 2004, Physics of the Solar Corona, Praxis (Berlin: Chichester and Springer-Verlag) 
Brueckner, G. E., Howard, R. A., Koomen, M. J., et al. 1995, Sol. Phys., 162, 357

Cargill, P. J., \& Priest, E. R. 1982, Sol. Phys., 76, 357

Forbes, T. G., \& Malherbe, J. M. 1986, ApJ, 302, L67

Forbes, T. G., \& Priest, E. R. 1983, Sol. Phys., 84, 169

Forbes, T. G., \& Priest, E. R. 1995, ApJ, 446, 377

Gallagher, P. T., Dennis, B. R., Krucker, S., Schwartz, R. A., \& Tolbert, A. K. 2002, Sol. Phys., 210, 341

Garcia, H. A. 1994, Sol. Phys., 154, 275

Handy, B. N., Acton, L. W., Kankelborg, C. C., et al. 1999, Sol. Phys., 187, 229 Hurford, G. J., Schmahl, E. J., Schwartz, R. A., et al. 2002, Sol. Phys., 210, 61

Karlický, M. 2004, New Astron., 9, 383

Kennewell, J. A., \& Cornelius, D. W. 1983, Aus. Phys., 20, 276

Kopp, R. A., \& Pneuman, G. W. 1976, Sol. Phys., 50, 85

Lin, J., \& Forbes, T. G. 2000, J. Geophys. Res., 105, 2375

Lin, R. P., Dennis, B. R., Hurford, G. Y., et al. 2002, Sol. Phys., 210, 3

McKenzie, D. E., \& Hudson, H. S. 1999, ApJ, 519, L93

Moore, R. L., McKenzie, D. L., Svestka, Z., et al. 1980, Solar Flares, ed. P. A. Sturrock (Boulder: Colorado Assoc. Univ. Press), 341
Moore, R. L., Sterling, A. C., Hudson, H. S., \& Lemen, J. R. 2001, ApJ, 552, 833

Nikajima, H., Nishio, M., Enome, S., et al. 1995, Proc. IEEE, 82, 705

Noglik, J. B., Walsh, R. W., \& Ireland, J. 2005, A\&A, 441, 353

Pneuman, G. W. 1981, Solar Flare Magnetohydrodynamics, ed. E. R. Priest (London: Gordon and Breach), 379

Romano, P., Zuccarello, F., \& Contarino, L. 2007, A\&A, 240, 49

Roussev, I. I., Forbes, T. G., Gombosi, T. I., et al. 2003, ApJ, 588, L45

Schmieder, B., Forbes, T. G., Malherbe, J. M., \& Machado, M. E. 1987, ApJ, 317,956

Sheeley, N. R., Warren, H. P., \& Wang, Y. M. 2004, ApJ, 66, 1224

Stähli, M., Gary, D. E., \& Hurford, G. J. 1989, Sol. Phys., 120, 351

Sturrock, P. A., Weber, M., Wheatland, M. S., \& Wolfson, R. 2001, ApJ, 548, 492

Sui, L., \& Holman, G. D. 2003, ApJ, 596, L251

Svestka, Z. 2007, Sol. Phys., 246, 393

Török, T., \& Kliem, B. 2005, ApJ, 630, L97

Veronig, A. M., \& Brown, J. 2004, ApJ, 603, L117 